\title{
Calcium Pyrophosphate Deposition Arthropathy (Pseudogout) Presenting in the Cervical Spine
}

Nathan Heckerson, MD, Connie Teng, MD

\section{Introduction}

Calcium pyrophosphate deposition (CPPD) arthropathy, more commonly known as pseudogout, is a rheumatologic disease caused by the proliferation of excess inorganic calcium pyrophosphate in the synovium. This is due to excessive breakdown of ATP leading to the accumulation of inorganic pyrophosphate which binds to calcium and deposits in cartilage and synovial tissue. The mechanism of crystal deposition in joint spaces is similar to gout, but the presentation usually resembles that of osteoarthritis or rheumatoid arthritis. The disease may present as an acute flare or may be asymptomatic and discovered incidentally on radiographs. This diagnosis is uncommon, but is becoming more prevalent as diagnostic techniques improve.

\section{Case Report}

A 74 year old female with a history of osteoarthritis presented with new-onset diplopia and six months of unilateral neck and occipital pain. On physical exam, she had bony deformities of the hands and feet consistent with her diagnosis of osteoarthritis, including Heberden and Bouchard nodes. Examination of her neck only revealed kyphosis. CT imaging of the cervical spine identified a mass encompassing the dens of the C2 vertebral body. MRI further characterized the mass as a pannus, raising suspicion for a rheumatologic etiology. Due to the risks of an invasive procedure, biopsy of the mass was not performed. A joint survey provided support for the diagnosis of CPPD arthropathy by demonstrating multiple areas of chondrocalcinosis. The patient was treated with daily colchicine and a short course of oral corticosteroids, showing some improvement.
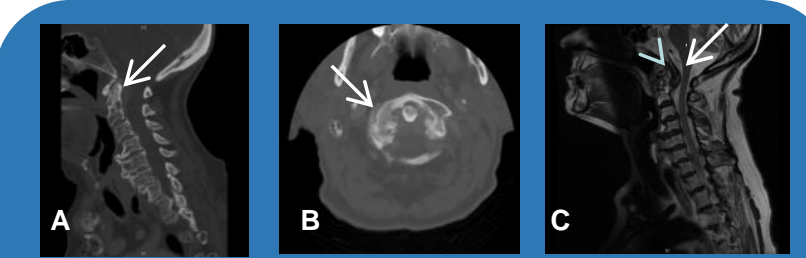

Figure 1: Sagittal (A) and axial (B) views from CT and sagittal view from MRI $(\mathrm{C})$ of the cervical spine, demonstrating a pannus at the $\mathrm{C} 2$ level.

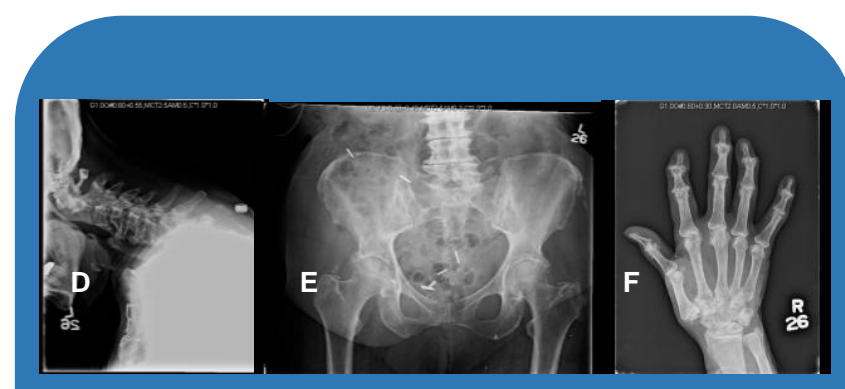

Figure 2: Multiple images from the joint survey representing findings consistent with a diagnosis of CPPD arthropathy, including hyperostosis and anterolisthesis of the cervical spine (D) and chondrocalcinosis of the hip joints (E) and the triangular ligament of the wrist $(\mathrm{F})$.

\section{References}

-Clin Exp Rheumatol. 2008 Nov-Dec;26(6):1040-6. -J Neurol Sci. 2010 Sep 15;296(1-2):79-82. Epub 2010 Jun 19.

-Primer on the Rheumatic Diseases. 2008. $13^{\text {th }}$ Ed. Springer.
Diagnostic Evaluation

A joint survey is the standard radiologic study. This usually focuses on the wrists, knees, hips, and pubic symphysis in an attempt to identify chondrocalcinosis and a typical pattern of erosive changes as seen in Figure 2.

Polarized microscopic examination of the joint fluid aspirate reveals weakly positive, rhomboid, birefringent crystals.

\section{Treatment}

Acute exacerbations are treated with systemic or intraarticular corticosteroids. Chronic disease management involves a combination of colchicine, NSAIDs, and low-dose intermittent steroids.

\section{Discussion}

Calcium pyrophosphate deposition disease often mimics the presentation of osteoarthritis or rheumatoid arthritis. It can cause both chronic degenerative changes and acute attacks making the diagnosis very difficult. It is relatively rare for CPPD arthropathy to involve the cervical spine. More commonly affected locations include the knees, hips, pubic symphysis, and wrists. In the case presented, the patient had been diagnosed with osteoarthritis in the past based on clinical findings, but later received a new diagnosis after undergoing more extensive testing. It is common for these patients to have multiple rheumatologic diagnoses, but this one is often missed if a full evaluation is not performed. 